\title{
Conhecimentos dos agentes comunitários de saúde sobre crack e outras drogas
}

\author{
Community health workers' knowledge about crack and other drugs \\ Conocimiento de los agentes comunitarios de salud acerca del crack y de otras drogas
}

Analine Fernandes. Universidade de Passo Fundo (UPF). Secretaria Municipal de Saúde de Passo Fundo. Passo Fundo, RS, Brasil. analinefernandes@yahoo.com.br (Autor correspondente)

Airton Tetelbom Stein. Fundação Universidade Federal de Ciências da Saúde de Porto Alegre (UFCSPA). Porto Alegre, RS, Brasil. airton.stein@gmail.com Camila Giugliani. Universidade Federal do Rio Grande do Sul (UFRGS). Porto Alegre, RS, Brasil. giugli@hotmail.com

\section{Resumo}

Objetivo: identificar os conhecimentos dos agentes comunitários de saúde (ACS), do município de Passo Fundo sobre o crack e outras drogas e descrever seus perfis sociodemográfico e de saúde. Métodos: estudo descritivo realizado com 78 ACS de Passo Fundo. A coleta de dados ocorreu por meio da aplicação de um questionário estruturado e autoaplicável sobre dados demográficos e de saúde dos ACS, e de um questionário de investigação dos conhecimentos sobre crack e demais drogas. Foram realizados análise descritiva, teste de Tukey e correlações lineares de Pearson e Spearman. Resultados: todos os ACS eram do sexo feminino, com idade média de 41,1土9,6 anos, sendo que 57,7\% delas possuíam 0 ensino médio completo e 69,2\% eram casadas. Além disso, 11,5\% eram tabagistas, 93,6\% negaram dependência ao álcool, e a maioria tinha dependentes químicos em sua área de abrangência. Metade delas apresentou problemas familiares. Em relação à saúde, 29,5\% obtiveram SRQ com pontuação sugerindo possibilidade de Transtornos Mentais Comuns, e 51,3\% afirmaram ser portadoras de doenças crônicas. A prova de conhecimentos obteve $34,5 \%$ de média de acertos, sendo o maior número de respostas corretas entre as ACS com maior escolaridade ( $p=0,006)$. Conclusão: 0 baixo número de acertos em teste de conhecimento evidencia uma necessidade urgente: a de reforçar a formação das ACS. 0 perfil específico dessas profissionais suscita a necessidade de maior atenção à sua saúde, bem como de novas pesquisas na área.

\begin{abstract}
Objective: to identify the knowledge of community health agents (CHA) of the city of Passo Fundo on crack and other drugs and to describe their sociodemographic and health profiles. Methods: descriptive study with $78 \mathrm{CHA}$ from Passo Fundo. Data collection occurred through the application of a structured and self-administered questionnaire on demographics and health of CHA, and another on knowledge about crack and other drugs. Descriptive analysis and Tukey's test, as well as Pearson's product moment correlation and Spearman's correlation were performed. Results: all CHA were female, mean age $41.1 \pm 9.6$ years, with $57.7 \%$ of them having completed high school and $69.2 \%$ being married. Also, $11.5 \%$ were smokers, 93.6\% denied alcohol dependence and most had drug addicts in their coverage area. Half of them presented family problems. In relation to health, 29.5\% obtained an SRQ score suggesting the possibility of Common Mental Disorders, and 51.3\% said that they had chronic diseases. In the knowledge test the average of correct answers was 34.5\%, with the highest number of correct answers being among the CHA with more schooling $(p=0.006)$. Conclusion: the low number of correct answers on the knowledge test shows an urgent need to strengthen the training of CHA. The specific profile of these professionals raises the need for more attention to their health, as well as new research in the area.
\end{abstract}

\section{Resumen}

Objetivo: identificar los conocimientos de los agentes comunitarios de salud (ACS), de la ciudad de Passo Fundo, en relación al crack y a otras drogas, y describir las características sociodemográficas y de salud. Métodos: estudio descriptivo con 78 ACS de Passo Fundo. Los datos fueron recolectados a través de la aplicación de un cuestionario estructurado y auto administrado sobre demografía y salud de los ACS, y de otro de investigación de los conocimientos sobre el crack y otras drogas. Análisis descriptivo y test de Tukey, así como correlación de Pearson y correlación de Spearman, fueron

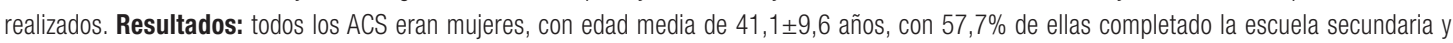
el $69,2 \%$ eran casadas. Además, el 11,5\% eran fumadoras, el 93,6\% negaron la dependencia del alcohol, y la mayoría era adicta a las drogas en su área de cobertura. La mitad de ellas presentaran problemas familiares. En relación a la salud, el 29,5\% obtuvieron un SRQ com puntuación que sugiere la posibilidad de los Trastornos Mentales Comunes, y el 51,3\% dijeron ser personas con enfermedades crónicas. La prueba de conocimientos alcanzó el 34,5\% promedio de respuestas correctas, con el mayor número de respuestas correctas entre las ACS con más educación $(p=0,006)$. Conclusión: el bajo número de respuestas correctas en la prueba de conocimiento muestra una necesidad urgente: fortalecer la capacitación de las ACS. El perfil específico de estas profesionales se plantea la necesidad de una mayor atención a su salud, así como la necessidad de nuevas investigaciones.

Como citar: Fernandes A, Stein AT, Giugliani C. Conhecimentos dos agentes comunitários de saúde sobre crack e outras drogas. Rev Bras Med Fam Comunidade. 2015;10(34):1-10. http://dx.doi.org/10.5712/rbmfc10(34)924
Palavras-chave:

Saúde Mental

Cocaína Crack

Saúde da Família

Agentes Comunitários de Saúde Conhecimento

Keywords:

Mental Health Crack Cocaine Family Health Community Health Workers Knowledge

\section{Palabras clave:}

Salud Mental.

Cocaína Crack

Salud de la Familia

Agentes Comunitarios de Salud Conocimiento

\section{Fonte de financiamento: declaram não haver. Parecer CEP: UPF 220/2012 03/12/2012. \\ Conflito de interesses: declaram não haver. Recebido em: 22/04/2014. Aprovado em: 16/12/2014.}




\section{Introdução}

Devido ao crescimento da prevalência de transtornos mentais em todo o mundo, em especial no que tange ao abuso de substâncias psicoativas, a Organização Mundial de Saúde (OMS) considera que seu manejo na Atenção Primária à Saúde (APS) é fundamental para facilitar o acesso dos pacientes aos serviços. ${ }^{1}$ A Estratégia Saúde da Família (ESF) é a principal forma de atuação da APS no Brasil, caracterizando-se pelo trabalho em equipes multiprofissionais e pela atuação na proximidade dos usuários, com potencial para a resolutividade das açôes. Tem, como interface entre o serviço de saúde e a comunidade, o Agente Comunitário de Saúde (ACS). 2,3

O uso abusivo e a dependência de drogas afetam milhares de pessoas, direta e indiretamente, gerando alta carga global de doenças, além de seus danos configurarem um problema de saúde pública por sua grande dimensão. Resultam em custos econômicos para o Sistema Único de Saúde (SUS) e custos sociais e pessoais para a população. ${ }^{1,4}$

Entre as substâncias de abuso, o crack tem apresentado aumento de consumo, no Brasil e demais países, com prejuízos imensuráveis. Essa dependência tem-se mostrado com baixo índice de abstinência no tratamento e com inúmeras recidivas ao uso, evidenciando a necessidade de acompanhamento longitudinal e integral pelas equipes de saúde. ${ }^{5}$

Ainda há uma grande heterogeneidade entre as diversas equipes de ESF no Brasil, pois as mesmas possuem profissionais atuantes que não têm nenhuma formação teórica padronizada em relação à drogadição, o que dificulta uma atenção coordenada, integral, continuada e resolutiva.

Além da ESF atuando na atenção básica, o Ministério da Saúde do Brasil tem inserido estratégias de enfrentamento ao crack e outras drogas por meio das redes de atenção psicossocial e dos serviços nos demais níveis de atenção à saúde, considerando uma necessidade urgente de açóes efetivas e articuladas em relação ao tema. ${ }^{6}$

O ACS é fundamental na equipe de atenção básica já que é o trabalhador que realiza o primeiro contato da equipe de saúde com a comunidade e possui condiçôes de identificar os riscos e as vulnerabilidades da população em seu território. ${ }^{7}$ Considera-se a ESF como contexto adequado para tratamento e acompanhamento de pacientes com problemas em relação ao uso de substâncias psicoativas. ${ }^{8,9}$

No que diz respeito ao ACS, artigos identificaram que os profissionais têm representaçôes sociais de estereótipos e preconceitos em relação aos usuários de drogas ${ }^{10} \mathrm{e}$ visão da drogadição como dimensão esquecida nas medidas preventivas. ${ }^{11}$ Outros estudos evidenciaram que os mesmos têm sentimentos e atitudes de maior aceitação em relação ao uso de drogas, porém, maior dificuldade de abordagem dos usuários, ${ }^{12}$ e relatam que os ACS afirmam não se sentirem capacitados para prestar assistência adequada em saúde mental. Uma pesquisa sobre capacitaçáo dos ACS em reduçáo de danos evidenciou a possibilidade de atuação desse profissional na área. ${ }^{13}$ Outro trabalho mostrou que existe atuação eficaz do ACS após capacitação em intervenção breve. ${ }^{14}$

Revisão sistemática, recentemente publicada, traz evidências de que o ACS pode ser efetivo em inúmeras ações na atenção básica, em especial na saúde materno-infantil. No entanto, existe ainda a necessidade de avaliar a utilidade do ACS na saúde mental, incluindo a atuação com drogadição. ${ }^{15}$

Avaliando a saúde mental do próprio ACS, tem-se identificado um elevado grau de ansiedade,${ }^{16}$ altas prevalências de transtornos mentais comuns e níveis moderados e altos de esgotamento profissional, ${ }^{17}$ o que evidencia que o sofrimento mental dos agentes pode estar relacionado ao trabalho. ${ }^{18}$

Nesse contexto, nossa pesquisa tem por objetivo identificar os conhecimentos dos agentes comunitários de saúde sobre crack e outras drogas, assim como as características sociodemográficas e de saúde mental desses profissionais.

\section{Métodos}

Trata-se de um estudo descritivo, realizado no município de Passo Fundo, no Norte do Rio Grande do Sul, localizado a 293 quilômetros de distância da capital do Estado. O município de $783,42 \mathrm{~km}^{2}$ possui, segundo dados do Instituto Brasileiro de Geografia e Estatística (IBGE) ${ }^{19}$ do ano de 2010, uma população de 184.826 habitantes, sendo a Secretaria Municipal de Saúde a responsável pela ordenação dos serviços de saúde. ${ }^{20}$

A população da pesquisa foi constituída por todos os ACS contratados no município de Passo Fundo e teve, como critério de inclusão, estar vinculado à ESF ou ao PACS e, como critério de exclusão, estar em licença-saúde no período da coleta de dados. 
Para a referida coleta, foram utilizados como instrumentos dois questionários autoaplicáveis identificados: um investigou o perfil sociodemográfico e de saúde dos ACS, e o outro, os conhecimentos dos mesmos sobre drogadição. A coleta ocorreu em data previamente agendada com todos os ACS, no auditório da Universidade de Passo Fundo, em horário de trabalho, sob orientação e supervisão da pesquisadora responsável.

Para caracterizar a população estudada, foi aplicado um questionário identificado, estruturado e autoaplicado de dados sociodemográficos contendo as seguintes variáveis: renda familiar, classe social de acordo com a Associação Brasileira de Empresas e Pesquisa (ABEP), ${ }^{21}$ idade, sexo, escolaridade, estado civil, número de filhos, religião, tempo de trabalho como ACS, vínculo empregatício, participação em conselho municipal de saúde (CMS), conselho distrital de saúde (CDS) ou grupo comunitário, capacitação sobre dependência química e outros temas oferecidos pela Secretaria Municipal de Saúde (SMS) ou afins, uso de medicamentos, presença de doenças crônicas, problemas relacionados a drogas na comunidade e na família, estado de saúde autorreferido e consumo de drogas do ACS.

Para a identificação de transtornos mentais comuns (TMC), foi utilizado o Self Reporting Questionnaire (SRQ-20), ${ }^{22}$ desenvolvido pela OMS para estudos de TMC, na APS e na comunidade, em países em desenvolvimento. No Brasil, o estudo da validação, realizado em São Paulo, estabeleceu o resultado 7-8 como ponto de corte. ${ }^{22}$

$\mathrm{O}$ questionário $\mathrm{CAGE}^{23}$ (acrônimo referente às suas quatro perguntas: Cut down, Annoyed by criticism, Guilty e Eye-opener) foi aplicado para avaliar a possibilidade de dependência de álcool. No Brasil, sua validação foi feita em 1983 por Masur e Monteiro, que encontraram uma sensibilidade de $88 \%$ e uma especificidade de $83 \% .{ }^{23}$ Resultados com dois pontos ou mais identificam grande possibilidade de dependência do álcool.

Para a análise dos conhecimentos prévios do ACS acerca de drogadição, realizou-se um questionário de conhecimentos com quarenta perguntas em relação ao crack e a outras drogas. Os conhecimentos abordados foram: definiçóes e classificação do uso de drogas; reconhecimento dos efeitos do uso e do abuso por meio de sinais e sintomas; critérios de risco; estágios de mudança e entrevista motivacional; encaminhamentos; orientaçôes sobre uso de medicamentos e rotinas de acompanhamento; reduçấo de danos; opçôes de tratamento; instrumentos de triagem; e prevenção e legislação. O questionário foi desenvolvido pelos pesquisadores do estudo e pela equipe do Viva Voz (Serviço Nacional de Orientaçóes e Informaçóes sobre a Prevenção do Uso de Drogas, sediado em Porto Alegre e sob a coordenação da Universidade Federal de Ciências da Saúde da mesma cidade), e adaptado pelo grupo de pesquisa para o público-alvo. Não foi encontrado, na literatura, nenhum outro instrumento destinado a medir conhecimento sobre drogadição; por esse motivo, adotou-se o referido questionário.

O teste piloto foi aplicado em dez ACS do município de Passo Fundo para avaliação do nível de entendimento dos questionários e adequaçôes, sendo também realizado com outros profissionais da ESF (médicos, enfermeiros e técnicos de enfermagem) com a finalidade de verificação do nível de dificuldade.

Após a codificação, os dados foram analisados no Programa SPSS versão 18.0. As variáveis categóricas foram expressas como frequência absoluta e relativa, e as numéricas contínuas, como média \pm desvio padrão. A análise de variância foi utilizada para avaliar associaçóes entre o número de erros, de acertos ou de respostas "não sei" com as seguintes variáveis: drogadição na família, vínculo com unidade básica de saúde (UBS) ou ESF, uso de medicamentos antidepressivos e benzodiazepínicos, doença crônica, estado civil, participaçôes em cursos de capacitação, Conselhos de Saúde e organizaçóes comunitárias, classe social, autopercepçáo de saúde e SRQ-20. Para comparações múltiplas, utilizou-se o teste de Tukey. A correlação linear de Pearson foi aplicada para avaliar associaçôes entre o número de erros no questionário e o SRQ-20, e a correlação de Spearman, para verificar o número de erros e acertos, a escolaridade e o tempo de trabalho como ACS.

Este estudo - em observância às diretrizes da Resolução 466/2012 do Conselho Nacional de Saúde do Ministério da Saúde para pesquisa envolvendo seres humanos - obteve aprovaçáo do Comitê de Ética em Pesquisa da Universidade de Passo Fundo (177/2012 e 220/2012), assim como autorização para seu desenvolvimento e concordância do participante com assinatura de termo de consentimento livre e esclarecido.

\section{Resultados}

Entre os ACS vinculados ao município de Passo Fundo, sete $(7,6 \%)$ foram excluídos por estar em licença-saúde no período da coleta de dados. Houve sete recusas $(8,2 \%)$, sendo que seis foram de uma única unidade de saúde.

A pesquisa foi realizada com um total de 78 participantes, com 58 deles vinculados à ESF, e o restante, ao PACS. Todos

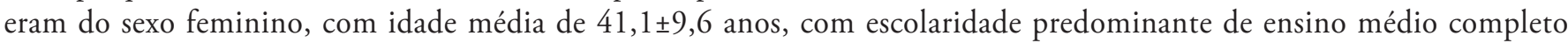
$(57,7 \%)$.Na sua maioria, as ACS eram casadas ou estavam morando junto com seus respectivos companheiros $(69,2 \%)$, 
com um a dois filhos $(62,9 \%)$, com maior número pertencente à religião católica $(65,4 \%)$. A renda familiar média relatada foi de $1814,10 \pm 873,50$ reais e, de acordo com a classificação da ABEP, ${ }^{21}$ as entrevistadas pertenciam às classes sociais $\mathrm{C}$ e $\mathrm{D}$ $(98,7 \%)$, conforme apresentado na Tabela 1.

\begin{tabular}{|c|c|}
\hline Variável & Estatística* \\
\hline \multicolumn{2}{|l|}{ Escolaridade $(n=77)$} \\
\hline Ensino fundamental incompleto & $1(1,3 \%)$ \\
\hline Ensino fundamental completo & $7(9,1 \%)$ \\
\hline Ensino médio incompleto & $15(19,5 \%)$ \\
\hline Ensino médio completo & $45(58,4 \%)$ \\
\hline Ensino superior incompleto & $6(7,8 \%)$ \\
\hline Ensino superior completo & $3(3,9 \%)$ \\
\hline \multicolumn{2}{|l|}{ Estado civil } \\
\hline Casada & $54(69,3 \%)$ \\
\hline Separada & $12(15,4 \%)$ \\
\hline Solteira & $9(11,5 \%)$ \\
\hline Viúva & $3(3,8 \%)$ \\
\hline \multicolumn{2}{|l|}{ Número de filhos } \\
\hline 0 & $6(7,7 \%)$ \\
\hline $1-2$ & $49(62,9 \%)$ \\
\hline 3 ou mais & $23(29,5 \%)$ \\
\hline \multicolumn{2}{|l|}{ Religião } \\
\hline Católica & $51(65,4 \%)$ \\
\hline Espírita & $2(2,6 \%)$ \\
\hline Evangélica & $20(25,6 \%)$ \\
\hline Outras religiões & $5(6,4 \%)$ \\
\hline \multicolumn{2}{|l|}{ Extrato social } \\
\hline C & $36(46,1 \%)$ \\
\hline $\mathrm{D}$ & $41(52,6 \%)$ \\
\hline$E$ & $1(1,3 \%)$ \\
\hline
\end{tabular}

Quanto ao perfil de trabalho das ACS, a maioria tinha vínculo com unidade de ESF (74,4\%), o tempo de trabalho médio relatado foi de 8,2 $\pm 3,4$ anos, sendo que a maior parte não integrava conselhos de saúde do município; porém, participavam de outras organizaçôes comunitárias $(53,8 \%)$, como associação de moradores, igreja local e grupos de mães e mulheres (Tabela 2).

A Tabela 2 também mostra que apenas $14,1 \%$ das agentes já haviam participado de capacitação cujo tema central era drogadiçáo, enquanto $67,7 \%$ receberam treinamentos em outras áreas específicas (sendo maior a prevalência de temas como hipertensão arterial sistêmica, diabetes mellitus, doenças sexualmente transmissíveis, vacinação, aleitamento materno entre outros). No entanto, quando interrogadas sobre sua percepção acerca de conhecimentos em drogadição, apenas $9 \%$ consideraram sua formação boa ou ótima, e 98,7\% referiram que uma capacitação nesse tema seria importante ou muito importante.

A Tabela 3 mostra dados sobre a situação de saúde em que as profissionais se encontravam. Quase metade das ACS referiu ser sedentária ou ter prática leve de atividade física, 11,5\% eram tabagistas, 93,6\% não apresentaram possibilidade de dependência de álcool e 51,3\% relataram ser portadoras de alguma doença crônica (hipertensão arterial sistêmica, depressão, ansiedade, lombalgia e diabete mellitus, em frequência decrescente).

No autorrelato da própria percepção de saúde, a maioria considerou estar em bom ou ótimo estado de saúde (69,2\%), mas $29,5 \%$ delas tinham SRQ elevado, evidenciando a possibilidade de transtornos mentais comuns. O escore médio, na escala SRQ-20, foi 4,8 3 3,9, e observaram-se escores $\geq 7$ em 23 (29,5\%) e $\geq 8$ em 15 (19,2\%). Em relação ao uso de medicamentos controlados, $25,6 \%$ relataram fazer uso de antidepressivos e $16,7 \%$, de benzodiazepínicos, com tempo de uso de 3,2 $\pm 2,4$ e $1,7 \pm 1,6$ anos, respectivamente (Tabela 3 ). 
Tabela 2. Variáveis relacionadas às características de trabalho das ACS de Passo Fundo (RS) em 2012 ( $\mathrm{n}=78$ ).

\begin{tabular}{|c|c|}
\hline Variável & Estatística* $^{\star}$ \\
\hline \multicolumn{2}{|l|}{ Local de vínculo } \\
\hline $\mathrm{ESF}^{\star \star}$ & $58(74,4 \%)$ \\
\hline UBS $* \star \star$ & $20(25,6 \%)$ \\
\hline Tempo de trabalho como ACS ${ }^{\#}$ (anos) & $8,2 \pm 3,4$ \\
\hline \multicolumn{2}{|l|}{ Participação no conselho de saúde } \\
\hline Municipal & $6(7,7 \%)$ \\
\hline Distrital & $9(11,5 \%)$ \\
\hline Nenhuma participação & $63(80,8 \%)$ \\
\hline \multicolumn{2}{|c|}{ Participação em organizações comunitárias } \\
\hline Sim & $42(53,8 \%)$ \\
\hline Não & $36(46,2 \%)$ \\
\hline \multicolumn{2}{|l|}{ Capacitação em drogadição } \\
\hline $\mathrm{SMS}^{+}$ & $7(9,0 \%)$ \\
\hline Externa & $4(5,1 \%)$ \\
\hline Nenhuma & $67(85,9 \%)$ \\
\hline \multicolumn{2}{|c|}{ Percepção quanto à formação em drogadição } \\
\hline Muito ruim - ruim & $37(47,4 \%)$ \\
\hline Razoável & $34(43,6 \%)$ \\
\hline Boa - ótima & $7(9,0 \%)$ \\
\hline \multicolumn{2}{|c|}{ Percepção quanto à necessidade de capacitação em drogadição } \\
\hline Desnecessária - pouco importante & $1(1,3 \%)$ \\
\hline Neutra & 0 \\
\hline Importante - muito importante & $77(98,7 \%)$ \\
\hline
\end{tabular}

Evidenciou-se a associação estatisticamente significativa entre autopercepção de saúde e $S R Q-20$ ( $F=6,48, d f=3, p=0,001)$. Observou-se que os indivíduos com autopercepção de saúde razoável apresentavam escores SRQ-20 significativamente maiores

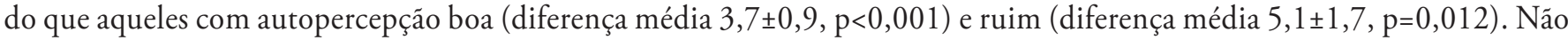
se observou diferença estatisticamente significativa quanto ao SRQ-20 entre os indivíduos com autopercepção de saúde boa e ótima (diferença média $1,4 \pm 1,6, \mathrm{p}=0,666$ ).

A Tabela 4 evidencia que 46 ACS (59\%) têm familiares que consomem drogas e 77 (98,7\%) atendem, na sua comunidade, indivíduos que consomem drogas. As substâncias mais utilizadas, segundo as agentes, são as drogas lícitas (álcool e tabaco) e, entre as ilícitas, o crack e a maconha, seguidas por cocaína, inalantes, anfetamina e heroína. Em relação ao consumo próprio de drogas, as ACS afirmaram fazer uso de cigarros e álcool (7,7\% e 3,8\%, respectivamente).

Quanto ao questionário de conhecimentos prévios das ACS sobre crack e outras drogas, a média de acertos foi de 34,5\%, com número médio de respostas corretas de $13,7 \pm 4,1$. O número médio de erros foi $17,1 \pm 5,7$, e o número médio de respostas "não sei responder" foi de $9,2 \pm 6,7$. As correlaçóes do número de acertos com as outras variáveis analisadas estáo descritas na Tabela 5.

Em relação ao número de acertos para conteúdos específicos sobre crack e outras drogas no questionário, identificou-se a média de 30,1\% em definiçóes e classificação do uso de drogas; $22,2 \%$ em reconhecimento dos efeitos do uso e do abuso por meio de sinais e sintomas apresentados pelos usuários; $14,5 \%$ em estágios de mudança do dependente químico e entrevista motivacional; $26,4 \%$ em encaminhamento para unidades de saúde e outros apoiadores; $28,2 \%$ em redução de danos; 20,5\% em opçôes de tratamento; 6,4\% em instrumentos de triagem e prevenção; e 23,8\% em legislação sobre drogadição.

Observou-se associação estatisticamente significativa entre escolaridade e número de acertos $\left(\mathrm{r}_{\mathrm{s}}=0,31, \mathrm{p}=0,007\right)$. Não se encontrou diferença estatística com relaçáo ao estado civil $(\mathrm{p}=0,783)$, à classe econômica $(\mathrm{F}=1, \mathrm{gl}=3, \mathrm{p}=0,396)$ e à idade $(\mathrm{r}=-0,02, \mathrm{p}=0,886)$ em relação ao número de acertos. 
Tabela 3. Variáveis sobre a saúde pessoal das ACS de Passo Fundo (RS) em 2012 (n=78).

\begin{tabular}{|c|c|}
\hline Variável & Estatística* \\
\hline \multicolumn{2}{|l|}{ Atividade física } \\
\hline Sedentária & $25(32,1 \%)$ \\
\hline Leve & $19(24,4 \%)$ \\
\hline Moderada & $24(30,8 \%)$ \\
\hline Intensa & $10(12,8 \%)$ \\
\hline \multicolumn{2}{|l|}{ Tabagismo } \\
\hline Não & $53(67,9 \%)$ \\
\hline Pregresso & $16(20,5 \%)$ \\
\hline Atual & $9(11,5 \%)$ \\
\hline Idade do início do tabagismo $(n=15)$ & $14,5 \pm 3,8$ \\
\hline Cigarros/dia* & $8,9 \pm 5,6$ \\
\hline Tentativa de cessação\# & $8(88,9 \%)$ \\
\hline \multicolumn{2}{|l|}{$\operatorname{CAGE}^{* *}(n=77)$} \\
\hline 0 & $73(94,8 \%)$ \\
\hline 1 & $2(2,6 \%)$ \\
\hline 2 & $2(2,6 \%)$ \\
\hline Doença crônica referida & $40(51,3 \%)$ \\
\hline DM & $8(10,3 \%)$ \\
\hline HAS & $22(28,2 \%)$ \\
\hline Dislipidemia & $8(10,2 \%)$ \\
\hline Depressão & $14(17,9 \%)$ \\
\hline Ansiedade & $12(15,4 \%)$ \\
\hline Convulsão & $1(1,3 \%)$ \\
\hline Hipotireoidismo & $1(1,3 \%)$ \\
\hline Lombalgia & $8(10,3 \%)$ \\
\hline Gastrite & $5(6,4 \%)$ \\
\hline Outro & $4(5,1 \%)$ \\
\hline \multicolumn{2}{|l|}{ Autopercepção de saúde } \\
\hline Muito ruim - ruim & $1(1,3 \%)$ \\
\hline Razoável & $23(29,5 \%)$ \\
\hline Boa - ótima & $54(69,2 \%)$ \\
\hline SRQ-20 & $4,8 \pm 3,9$ \\
\hline$\geq 7$ & $23(29,5 \%)$ \\
\hline$\geq 8$ & $15(19,2 \%)$ \\
\hline Uso de antidepressivo & $20(25,6 \%)$ \\
\hline Tempo de uso de antidepressivo em anos $(n=19)$ & $3,2 \pm 2,4$ \\
\hline Uso de Benzodiazepínico & $13(16,7 \%)$ \\
\hline Tempo de uso de BZD*** em anos $(n=12)$ & $1,7 \pm 1,6$ \\
\hline
\end{tabular}

Tabela 4. Consumo de substâncias psicoativas das ACS de Passo Fundo (RS) em 2012*.

\begin{tabular}{lccc}
\hline & \multicolumn{3}{c}{ Grupo } \\
\cline { 2 - 4 } & Familiar $(\mathrm{n}=78)$ & Comunidade $(\mathrm{n}=78)$ & ACS $(\mathrm{n}=77)$ \\
\hline Consumo de drogas & $46(59,0 \%)$ & $77(98,7 \%)$ & $9(11,5 \%)$ \\
Álcool & $32(41,0 \%)$ & $73(93,6 \%)$ & $3(3,8 \%)$ \\
Cigarro & $35(44,9 \%)$ & $71(91,0 \%)$ & $6(7,7 \%)$ \\
Cocaína & $2(2,6 \%)$ & $32(41,0 \%)$ & - \\
Crack & $14(17,9 \%)$ & $64(82,1 \%)$ & - \\
Inalante & - & $8(10,3 \%)$ & - \\
Anfetamina & - & $2(2,6 \%)$ & - \\
Heroína & - & $1(1,3 \%)$ & - \\
Maconha & $7(9,0 \%)$ & $49(62,8 \%)$ & - \\
Êxtase & - & - & - \\
LSD & - & - & - \\
\hline
\end{tabular}

${ }^{*}$ Os valores expressam a frequência absoluta e relativa. 
Tabela 5. Associação entre número de acertos e demais variáveis das ACS de Passo Fundo (RS) em 2012.

\begin{tabular}{|c|c|}
\hline Variável & Número de acertos $p^{\star}$ \\
\hline Escolaridade & $p=0,006$ \\
\hline Ensino fundamental/médio incompleto & $11,6 \pm 3,9$ \\
\hline Ensino médio completo & $14,8 \pm 3,4$ \\
\hline Ensino superior & $16,8 \pm 3,1$ \\
\hline Classe social (APEB) & $p=0,507$ \\
\hline$C 1(n=13)$ & $15,1 \pm 4,7$ \\
\hline $\mathrm{C} 2(\mathrm{n}=23)$ & $12,8 \pm 5,0$ \\
\hline$D(n=41)$ & $13,8 \pm 3,4$ \\
\hline$E(n=1)$ & 11,0 \\
\hline Estado Civil & $p=0,783$ \\
\hline Solteira $(n=9)$ & $14,3 \pm 4,2$ \\
\hline Casada $(n=54)$ & $13,8 \pm 4,4$ \\
\hline Separada $(n=12)$ & $12,8 \pm 3,2$ \\
\hline Viúva $(n=3)$ & $12,3 \pm 1,5$ \\
\hline Vínculo & $p=0,788$ \\
\hline UBS & $13,5 \pm 3,7$ \\
\hline ESF & $13,7 \pm 4,3$ \\
\hline Participação no CMS & $p=0,603$ \\
\hline Não (n=71) & $13,8 \pm 4,1$ \\
\hline $\operatorname{Sim}(n=6)$ & $12,8 \pm 5,4$ \\
\hline Participação no CDS & $p=0,268$ \\
\hline Não (n=69) & $13,5 \pm 4,1$ \\
\hline $\operatorname{Sim}(n=9)$ & $15,1 \pm 4,5$ \\
\hline Participação em organizações comunitárias & $p=0,445$ \\
\hline Não $(n=36)$ & $13,3 \pm 4,0$ \\
\hline $\operatorname{Sim}(n=42)$ & $14,0 \pm 4,3$ \\
\hline Capacitação SMS & $p=0,874$ \\
\hline Não (n=71) & $13,7 \pm 4,0$ \\
\hline $\operatorname{Sim}(n=7)$ & $13,4 \pm 5,9$ \\
\hline Capacitação externa & $p=0,149$ \\
\hline Não (n=74) & $13,8 \pm 4,0$ \\
\hline $\operatorname{Sim}(n=4)$ & $10,8 \pm 4,7$ \\
\hline Doença crônica & $p=0,223$ \\
\hline Não (n=38) & $13,1 \pm 4,3$ \\
\hline $\operatorname{Sim}(n=40)$ & $14,2 \pm 4,0$ \\
\hline Uso de antidepressivo & $p=0,507$ \\
\hline Não (n=58) & $13,5 \pm 4,3$ \\
\hline $\operatorname{Sim}(n=20)$ & $14,2 \pm 3,8$ \\
\hline Uso de benzodiazepínico & $p=0,284$ \\
\hline Não (n=65) & $13,9 \pm 4,3$ \\
\hline $\operatorname{Sim}(n=13)$ & $12,5 \pm 3,1$ \\
\hline Drogadição na família & $p=0,260$ \\
\hline Não & $13,0 \pm 4,8$ \\
\hline Sim & $14,1 \pm 3,6$ \\
\hline
\end{tabular}

*0s valores expressam a frequência absoluta e relativa ou a média \pm desvio padrão.

O número médio de respostas corretas entre as agentes vinculadas à UBS foi de $13,5 \pm 3,7$, e, entre aquelas vinculadas à ESF, de $13,7 \pm 4,3, p=0,788$. Não se observou associação estatisticamente significativa entre tempo de trabalho como ACS e número de acertos $\left(\mathrm{r}_{\mathrm{s}}=-0,15, \mathrm{p}=0,182\right)$. As participantes que integram conselhos de saúde e outras participaçôes comunitárias não apresentaram diferença importante no número de acertos. As que participaram de capacitaçóes em drogadição (da secretaria ou externas) não obtiveram resultados estatisticamente significativos entre elas ( $\mathrm{p}=0,874$ e 0,149 ). 
As agentes que referiram ser portadoras de doenças crônicas tiveram semelhante número de acertos quando comparadas com as que se definiram como hígidas $(\mathrm{p}=0,223)$. Não houve a associação estatisticamente significativa entre SRQ-20 e número de acertos $(\mathrm{r}=0,03, \mathrm{p}=0,772)$, tampouco entre número de acertos e uso de medicamento antidepressivo $(\mathrm{p}=0,507)$ e benzodiazepínico $(\mathrm{p}=0,284)$.

Não se observou associação estatisticamente significativa entre número de acertos e drogadição na família da ACS. Aquelas que relataram drogadição na família acertaram em média 14,1 33,6 questôes, ao passo que, entre aquelas que negaram, o número médio de acertos foi de $13 \pm 4,8, \mathrm{p}=0,260$.

\section{Discussão}

O perfil dos ACS foi composto por mulheres em sua totalidade, resultado que vai ao encontro da maioria das publicaçóes, em que as mulheres têm predominância na funçáo de ACS e na prestação de cuidados no Brasil. Estas se encontram, em maior parte, casadas, sendo adultas jovens com escolaridade média a baixa e renda familiar semelhante ao encontrado em outros estudos que avaliaram ACS. . $^{1,11,12,13,14,15,16}$

A grande maioria das ACS nunca havia realizado capacitação sobre substâncias psicoativas, fato que pode estar relacionado com o mau desempenho no teste de conhecimentos. Aquelas com maior escolaridade obtiveram maior número de acertos acerca da drogadição. A dependência química tem tido crescimento alarmante no Brasil e no mundo e, mesmo com prevalência menor do que algumas doenças crônicas, tem causado prejuízos sociais, financeiros e humanos imensuráveis. ${ }^{1,4} \mathrm{~A}$ formação para agentes de saúde em áreas específicas da saúde mental, como a redução de danos ${ }^{13} \mathrm{e}$ a intervenção breve, ${ }^{14}$ demonstrou que os ACS tem bom aprendizado se submetidos à atualização adequada.

As ACS de Passo Fundo têm elevado contato com usuários de drogas e enfrentam em sua prática diária problemas relacionados à dependência, sendo que mais da metade tem familiares envolvidos, e quase a totalidade possui, em sua área de abrangência, algum caso. Para enfrentar essa realidade, uma minoria considerou que possui conhecimento sobre o assunto. Tal percepção foi confirmada com o teste de análise de conhecimentos específicos, com resposta correta em apenas um terço das questôes. O estudo sobre conhecimento dos ACS moradores de Marília (São Paulo) sobre o envelhecimento relatou um baixo índice de capacitação no assunto e, no teste de conhecimentos, a média de acertos foi de $40 \%,{ }^{24}$ porcentagem semelhante de acertos na investigação de conhecimentos em drogadição apresentada neste estudo. $\mathrm{O}$ bom resultado em capacitação para ACS também foi relatado em estudo sobre intervenção educativa em aleitamento materno, em que houve um aumento do escore médio de conhecimentos classificados como bons após a formação específica no assunto. ${ }^{25}$

As pesquisas já realizadas evidenciam que o tema drogadição ainda tem um estigma entre os profissionais da saúde, incluindo os ACS, com sentimentos de preconceito em relação aos usuários. ${ }^{10,11,12} \mathrm{Um}$ maior conhecimento sobre o assunto mostra-se urgente no momento em que esses profissionais estấo inseridos em ambientes de trabalho com elevada prevalência de pacientes e familiares com problemas relacionados à dependência química.

Entre as ACS, foram identificados elevado consumo de tabaco e pequena possibilidade de dependência de álcool. Metade das profissionais apresentou, no momento do estudo, alguma doença crônica, um quarto estava utilizando antidepressivos e um quinto, benzodiazepínico. Esse resultado revela que as próprias profissionais estão em sofrimento mental e com a saúde fragilizada, necessitando de cuidados. Além de maior atenção aos profissionais, a melhora de conhecimento sobre o tema em questáo pode diminuir o sofrimento no trabalho, oferecendo ao ACS mais autonomia e confiança. ${ }^{13}$

No estudo, metade das ACS referiu ser sedentária ou praticar atividade física leve. A pesquisa divulgada em $2009^{26}$ que analisou a prevalência de sedentarismo em profissionais da saúde evidenciou sedentarismo em $26,4 \%$ das profissionais de saúde do sexo feminino, sendo 14,9\% nas ACS. Nesse sentido, urge a necessidade de estratégias para estimular os profissionais de saúde a praticarem mais atividade física, proporcionando melhora da qualidade de vida do ACS e de toda a equipe de saúde.

Quanto à análise de transtornos mentais comuns com o SRQ, 29,5\% das profissionais apresentaram um escore sugestivo de transtornos mentais comuns, resultado com associação estatisticamente significativa se comparado com a própria autopercepção de saúde, em que as mesmas apresentaram resultado de teste compatível com o que percebem sobre sua saúde. Silva ${ }^{17}$ também aplicou o SRQ-20, mesmo instrumento utilizado no presente estudo, e encontrou, em agentes de saúde de São Paulo, uma prevalência de casos de transtornos mentais comuns de 43,3\%, valor mais elevado do que em Passo Fundo. No entanto, as diferenças regionais e de estilos de vida precisam ser consideradas. 
A investigação com outro instrumento para pesquisar ansiedade foi realizada por Resende em Uberlândia ${ }^{16}$ e demonstrou que a maioria dos ACS apresenta grau de ansiedade moderado, e uma parcela, grave. Destaca-se que instrumentos diferentes encontram resultados semelhantes na detecção de sofrimento mental do trabalhador da saúde.

Os ACS atuam frequentemente em condiçóes adversas, residindo em seu local de atuação laboral, com contato permanente com o foco de seu trabalho, geralmente em áreas com elevada frequência de uso de drogas, baixo poder aquisitivo e poucos recursos sociais. Para um melhor enfrentamento dessa realidade, faz-se necessária a formação adequada, considerando os conhecimentos populares dos ACS, com especial atenção ao trabalhador, constituindo redes de apoio social e em saúde.

O presente estudo náo avaliou os conhecimentos de todos os profissionais da equipe, mas sim daqueles com menor escolaridade e que convivem diariamente com os pacientes em seus domicílios, sem ter um curso técnico de formação para sua atividade profissional e sem o respaldo de um conselho de ética que regulamente sua atuação.

Aquelas ACS em afastamento do trabalho por motivo de licença-saúde podem estar afastadas por TMC ou por outras causas de doenças agudas ou crônicas que não foram avaliadas no estudo. De acordo com a coordenação da SMS, a maior parte dos desligamentos são por transtornos depressivos. As ACS que recusaram participar do estudo podem ter perfil sociodemográfico e de saúde, além de conhecimentos, diferentes dos das demais participantes.

A identificação dos questionários pode ser uma limitação do estudo, pois é capaz de restringir o grau de informação, principalmente em relação aos problemas de saúde pessoais e ao uso de drogas.

\section{Conclusão}

Existe uma lacuna na formaçáo das ACS, neste estudo relacionada à temática crack e outras drogas. O conhecimento que as ACS têm sobre o assunto mostrou-se pobre e enfocado em percepções gerais, sem instrumentalização técnica. Tal resultado é inquietante, pois a preparação técnica e ética deve ser constantemente abordada junto dos profissionais que atuam na linha de frente. Evidencia-se, assim, a necessidade de um programa de educação permanente nas equipes que inclua o tema da drogadiçáo e definição das atribuiçôes do ACS, assim como a garantia de atendimento nas unidades de saúde para os pacientes encaminhados pelos ACS.

O ACS é o elo entre a equipe e a comunidade. Essa atividade é de grande complexidade, em especial devido ao fato de esse profissional não ter uma formação específica, que abranja aspectos éticos e de drogadição. Como o ACS é parte da comunidade, muitas vezes vivencia os mesmos problemas, o que às vezes pode dificultar o cuidado.

A elevada prevalência de transtornos mentais em profissionais da saúde traz para a discussão um tema preocupante que necessita açóes urgentes, pois o ACS precisa estar em bom estado de saúde para prestar um cuidado com efetividade. O estado de saúde mental do ACS pode influenciar a relaçáa estabelecida com o usuário de drogas, prejudicando a atenção aos pacientes.

O estudo fornece informaçóes importantes para o planejamento de açôes de educação permanente e evidencia a necessidade de se intensificar o processo educativo entre os ACS. Profissionais capacitados podem produzir, em conjunto com os outros profissionais de saúde, uma melhoria na qualidade da atenção prestada à população, com consequente impacto sobre morbimortalidade, prevenção e tratamento do uso de drogas.

\section{Referências}

1. Organização Mundial de Saúde. Relatório de saúde mental e desenvolvimento. 2010. Saúde mental e desenvolvimento. Um problema emergente: Integração Mental. OMS: 2011.

2. Ministério da Saúde (BR). Saúde da Família: uma estratégia para a reorientação do modelo assistencial. Brasília, DF: Ministério da Saúde; 1997.

3. Ministério da Saúde (BR), Secretaria de Atenção à Saúde, Departamento de Atenção Básica. Política Nacional de Atenção Básica. $4^{a}$ ed. Brasília, DF: Ministério da Saúde; 2007.

4. Ministério da Saúde (BR); Presidência da República, Secretaria Nacional de Políticas sobre Drogas. Relatório brasileiro sobre drogas.Brasília, DF: IME USP; 2009.

5. Pulcherio G, Stolf AR, Pettenon M, Fenstrseifer DP, Kessler F. Crack-da pedra ao tratamento. Rev AMRIGS. 2010;54(3):337-343.

6. Ministério da Saúde (BR). Portaria nº 3088 de 23 de dezembro de 2011. Institui a Rede de Atenção Psicossocial (RAPS). Brasil: MS; 2011.

7. Ministério da Saúde (BR). Secretaria de Atenção à Saúde, Departamento de Atenção Básica. Guia prático do agente comunitário de saúde. Brasília: Ministério da Saúde; 2009. 
8. Andrade TM. Reflexões sobre políticas de drogas no Brasil. Ciênc Saúde Coletiva. 2011;16(12):4665-4674. http://dx.doi.org/10.1590/S1413-81232011001300015

9. Mângia EF, Barros JO. Projetos Terapêuticos e serviços de saúde mental: caminhos para a construção de novas tecnologias de cuidado. Rev Ter Ocup. São Paulo. 2009;20(2):85-91.

10. Oliveira JF, McCallum CA, Costa COG. Representações sociais dos agentes comunitários de saúde acerca do consumo de drogas. Rev Esc Enferm USP. 2010;44(3):611-8. http://dx.doi.org/10.1590/S0080-62342010000300009

11. Medeiros JS. Representações sociais dos profissionais do Programa Saúde da Família sobre o uso de drogas psicoativas no município de Fortaleza [dissertação]. Fortaleza: Universidade Federal do Ceará; 2006.

12. Barros MA, Pillon SC. Atitudes dos profissionais do Programa Saúde da Família diante do uso e abuso de drogas. Esc Anna Nery Rev Enferm. 2007;11(4):655-62. http://dx.doi.org/10.1590/S1414-81452007000400016

13. Nardi $H C$, Kroeff $L R$, Rigoni RQ. Sensibilização e capacitação em redução de danos: considerações dos agentes comunitários de saúde. Rev Psicolog Saúde. 2010;2(2):21-30.

14. Silva CJ. Impacto de um curso em diagnóstico e tratamento do uso nocivo e dependência do álcool sobre a atitude e conhecimento de profissionais da rede de atenção primária a saúde [tese]. São Paulo: Escola Paulista de Medicina da Universidade Federal de São Pauo; 2005.

15. Giugliani C, Harzheim E, Duncan MS, Duncan BB. Effectiveness of community health workers in Brazil: a systematic review j ambulatory care manage. 2011;34(4):326-338. http://dx.doi.org/10.1097/JAC.0b013e31822cbdfd

16. Resende MC, Azevedo RGS, Lourenço LR, Faria LS, Alves NF, Farina NP, et al. Saúde mental e ansiedade em agentes comunitários que atuam em saúde da família em Uberlândia (MG, Brasil). Ciênc Saúde Coletiva. 2011;16(4):2115-2121. http://dx.doi.org/10.1590/S1413-81232011000400011

17. Silva ATC, Menezes PR. Esgotamento profissional e transtornos mentais comuns em agentes comunitários de saúde. Rev Saúde Pública. 2008;42(5):921-9. http://dx.doi.org/10.1590/S0034-89102008000500019

18. Camelo SHH, Galon T, Marziale MHP. Formas de adoecimento pelo trabalho dos agentes comunitários de saúde e estratégias de gerenciamento. Rev Enferm UERJ. 2012 Dez;20(esp1):661-7.

19. Instituto Brasileiro de Geografia e Estatística (BR). Censo demográfico 2010. Rio de Janeiro: IBGE; 2010 [acesso em: 2012 May 8]. Disponível em: http://www.ibge.gov.br/cidadesat/topwindow.html

20. Prefeitura Municipal de Passo Fundo. Passo Fundo; 2012 [acesso em: 2012 May 9]. Disponível em: http://www.pmpf.rs.gov.br/secao. php? $=172 \& a=2 \& p m=1212$

21. Associação Brasileira de Empresas de Pesquisa (ABEP). Disponível em: http://www.abep.org. [acesso em 2012 Oct 08].

22. Gonçalves DM, Stein AT, Kapczinski F. Avaliação de desempenho do Self-Reporting Questionnaire como instrumento de rastreamento psiquiátrico: um estudo comparativo com o Structured Clinical Interview for DSM-IV-TR. Cad Saúde Pública. Rio de Janeiro. 2008;24(2):380-390. http://dx.doi.org/10.1590/S0102-311X2008000200017

23. Masur J, Monteiro M. Validation of the CAGE alcoholism screening test in Brazilian Psychiatry Inpatient Hospital Setting. Braz J Med Biol Res. 1983;16:215-8. Disponível em: http://europepmc.org/abstract/MED/6652293/reload=0;jsessionid=TI4YemOonpsITaMoNg1q.16

24. Ferreira VM, Ruiz T. Atitudes e conhecimentos de agentes comunitários de saúde e suas relações com idosos. Rev Saúde Pública. 2012;46(5):843-49. http://dx.doi.org/10.1590/S0034-89102012000500011

25. Machado MCHS, Oliveira JS, Parada CMGL, Venâncio SI, Tonete VLP, Carvalhaes MABL. Avaliação de intervenção educativa sobre aleitamento materno dirigido às agentes comunitárias de saúde. Rev Bras Saúde Matern Infant. 2010;10(4):459-468. http://dx.doi.org/10.1590/S1519-38292010000400006

26. Siqueira FCV, Nahas MV, Facchini LA, Piccini RX, Tomasi E, Thumé E et al. Atividade física em profissionais de saúde do Sul e Nordeste do Brasil. Cad Saúde Pública. 2009;25(9):1917-1928. http://dx.doi.org/10.1590/S0102-311X2009000900006 